\title{
Addendum to the Acknowledgements: How Online Communities of People With Long-Term Conditions Function and Evolve: Network Analysis of the Structure and Dynamics of the Asthma UK and British Lung Foundation Online Communities
}

Sagar Joglekar ${ }^{1}, \mathrm{MS}$; Nishanth Sastry ${ }^{1}$, PhD; Neil S Coulson ${ }^{2}$, PhD; Stephanie JC Taylor ${ }^{3}$, MD; Anita Patel ${ }^{3}$, PhD; Robbie Duschinsky ${ }^{4}$, PhD; Amrutha Anand ${ }^{5}$, MPH; Matt Jameson Evans ${ }^{5}$, MB BS; Chris J Griffiths ${ }^{3}$, D Phil; Aziz Sheikh $^{6}, \mathrm{MD}$; Pietro Panzarasa ${ }^{7}, \mathrm{PhD} ;$ Anna De Simoni ${ }^{3}, \mathrm{PhD}$

${ }^{1}$ Department of Informatics, King's College London, London, United Kingdom

${ }^{2}$ School of Medicine, University of Nottingham, Nottingham, United Kingdom

${ }^{3}$ Asthma UK Centre for Applied Research, Barts Institute of Population Health Sciences, Queen Mary University of London, London, United Kingdom

${ }^{4}$ Primary Care Unit, Department of Public Health and Primary Care, University of Cambridge, Cambridge, United Kingdom

${ }^{5}$ HealthUnlocked, London, United Kingdom

${ }^{6}$ Asthma UK Centre for Applied Research, Usher Institute of Population Sciences and Informatics, University of Edinburgh, Edinburgh, United Kingdom

${ }^{7}$ School of Business and Management, Queen Mary University of London, London, United Kingdom

Corresponding Author:

Anna De Simoni, PhD

Asthma UK Centre for Applied Research

Barts Institute of Population Health Sciences

Queen Mary University of London

58 Turner Street

London, E1 2AB

United Kingdom

Phone: 442078822520

Fax: 442078822

Email: a.desimoni@qmul.ac.uk

\section{Related Article:}

Correction of: http://www.jmir.org/2018/7/e238/

(J Med Internet Res 2018;20(9):e11564) doi: 10.2196/11564

The authors of "How Online Communities of People With Long-Term Conditions Function and Evolve: Network Analysis of the Structure and Dynamics of the Asthma UK and British Lung Foundation Online Communities" (J Med Internet Res 2018;20(7):e238) wish to add the following sentence to the Acknowledgments:

Nishanth Sastry was partially supported by the Grant No. ES/M00354X/1 (A Shared Space and a Space for
Sharing: A Transdisciplinary Exploration of Online Trust and Empathy).

The correction will appear in the online version of the paper on the JMIR website on September 4, 2018, together with the publication of this correction notice. Because this was made after submission to PubMed, Pubmed Central, and other full-text repositories, the corrected article also has been re-submitted to those repositories. 
Edited by G Eysenbach; this is a non-peer-reviewed article. Submitted 18.07.18; accepted 20.08.18; published 04.09.18.

Please cite as:

Joglekar S, Sastry N, Coulson NS, Taylor SJC, Patel A, Duschinsky R, Anand A, Jameson Evans M, Griffiths CJ, Sheikh A, Panzarasa

$P$, De Simoni A

Addendum to the Acknowledgements: How Online Communities of People With Long-Term Conditions Function and Evolve: Network

Analysis of the Structure and Dynamics of the Asthma UK and British Lung Foundation Online Communities

J Med Internet Res 2018;20(9):e11564

URL: http://www.jmir.org/2018/9/e11564/

doi: $\underline{10.2196 / 11564}$

PMID: $\underline{30578240}$

(C) Sagar Joglekar, Nishanth Sastry, Neil S Coulson, Stephanie JC Taylor, Anita Patel, Robbie Duschinsky, Amrutha Anand, Matt Jameson Evans, Chris J Griffiths, Aziz Sheikh, Pietro Panzarasa, Anna De Simoni. Originally published in the Journal of Medical Internet Research (http://www.jmir.org), 04.09.2018. This is an open-access article distributed under the terms of the Creative Commons Attribution License (https://creativecommons.org/licenses/by/4.0/), which permits unrestricted use, distribution, and reproduction in any medium, provided the original work, first published in the Journal of Medical Internet Research, is properly cited. The complete bibliographic information, a link to the original publication on http://www.jmir.org/, as well as this copyright and license information must be included. 